\title{
Job Rotation: An Effective Tool to Transfer the Tacit Knowledge within an Enterprise
}

\author{
Honglei Lu, Congjie Yang \\ School of Management, Jinan University, Guangzhou, China \\ Email: lisa900626@126.com
}

Received 20 February 2015; accepted 16 March 2015; published 17 March 2015

Copyright (C) 2015 by authors and Scientific Research Publishing Inc.

This work is licensed under the Creative Commons Attribution International License (CC BY). http://creativecommons.org/licenses/by/4.0/

(c) ()

\begin{abstract}
There are a variety of ways to transfer the tacit knowledge within an enterprise, and job rotation is one of the effective tools. This article reveals the three-stage process enterprises transferring the tacit knowledge by implementing job rotation, and analyses the efficiency of tacit knowledge transferring among individuals in different job rotation situations, as well as the mechanism of knowledge transforming from individual knowledge to job knowledge. At last, a mechanism to manage tacit knowledge transferring by implementing job rotation is constructed, including specific targeting sub-mechanism, dynamic sub-mechanism and feedback sub-mechanism. This article will help companies control the job rotation process, optimize the management of tacit knowledge, and achieve the organization's strategy objectives.
\end{abstract}

\section{Keywords}

Knowledge Management, Job Rotation, Tacit Knowledge Transfer, Management Mechanism

\section{Introduction}

Under the background of the era of knowledge economy, knowledge has become one of the most valuable strategic resources. Polanyi divided knowledge into explicit knowledge and tacit knowledge. Explicit knowledge is the knowledge that people can encode and disseminate effectively; tacit knowledge is the knowledge that people know, but it's difficult to express [1]. Tacit knowledge is difficult to be imitated or replicated by competitors, so that it owns higher strategic value for businesses. Tacit knowledge within the enterprise contains the individual's know-how, experience, insight, collaboration of group members, group culture, a common corporate vision, organizational routines and so on. The tacit knowledge can be further divided into "pseudo tacit knowledge" and "true tacit knowledge" [2]. Pseudo tacit knowledge is the knowledge which remains tacit due to the high cost to be expressed; true tacit knowledge is the knowledge which cannot be expressed due to the knowledge form and 
its attribute of inseparability from body [3]. The enterprises can achieve the transmission and creativity of internal knowledge only if the tacit knowledge can be transferred continuously, and then format the corporate intellectual capital which brings a sustainable competitive advantage to the enterprise [4].

Knowledge transfer is a process that the knowledge is transferred from knowledge sources to knowledge receptors [5]. Formal tacit knowledge transfer channels contain mentoring, group learning [6], establishing the enterprise internal knowledge market [7], expert database, knowledge map [8], etc. Some scholars put forward that the enterprise can also transfer tacit knowledge through informal communication channels, such as irregular seminars, coffee gap chatting and so on. In recent years, people gradually realize that job rotation is also an effective tool to realize tacit knowledge transferring within enterprises. Implementing job rotation provides the rotators with a real learning situation; at the same time, the knowledge receiver will be moved to the knowledge center to accelerate the two-way tacit knowledge transfer [9]. Then, what is the process that tacit knowledge is transferred through job rotation? Establishing tacit knowledge management mechanism, including target submechanism, dynamic sub-mechanism and feedback sub-mechanism, can realize tacit knowledge transfer process to support the realization of strategic goals. Then, how can we build the tacit knowledge management mechanism within an enterprise?

\section{The Process Using Job Rotation to Realize Tacit Knowledge Transfer}

The ultimate goal of enterprise tacit knowledge transfer is to ensure that the employees in the key position own sufficient knowledge to qualify the positions, so that they can fully support the key business processes of the enterprise, and further to realize the short term and long term strategic goals. Using job rotation to achieve enterprise tacit knowledge transfer process includes three phases:

\subsection{The Stage to Identify the Tacit Knowledge Transfer Subjects}

To identify the tacit knowledge transfer subjects is the origin of tacit knowledge transfer process, generally contains four steps. 1) According to enterprise's strategic development target, positioning key positions as knowledge receiving jobs. Enterprise strategic objective is based on the core business processes, so that to identify the key positions in the core business process and to achieve the goals of these jobs will directly decides the realization of enterprise strategic target. 2) Comparing the tacit knowledge gap between the qualification of key positions and the existing incumbents, and determine the tacit knowledge transfer requirements. Use competency analysis method to analyze key positions, and identify the necessary tacit knowledge which the core positions require and then to measure the tacit knowledge stock of the incumbents to analysis the tacit knowledge gap between the incumbents and job qualifications, as well as the demand for knowledge transfer. Measurement tools and methods could be chose depending on the type of tacit knowledge, for example, the tacit knowledge with low implicit levels (e.g. professional skills, problem solving, etc.) can be measured with evaluate scale; For the tacit knowledge with high degree implicit (e.g. individual mental models) are generally measured with the scene view method, experiment method, role playing method, man-machine auxiliary experiment. 3) According to the demand of the tacit knowledge transfer, to position the knowledge outputting positions as target rotation job. Utilize the knowledge map or job analysis to position the target outputting positions which own the required knowledge. If the enterprise need to transfer the knowledge stored in an individual, then we need to position knowledge carrier person as the primary knowledge transmitter. 4) To select rotators. Enterprises select the right person in the knowledge receiving positions to rotate to the knowledge outputting positions in order get new tacit knowledge. This requires rotators have subjective willingness to participate in job rotation and have good learning ability.

\subsection{Tacit Knowledge Transfer Stage}

After identify the tacit knowledge transfer subject, the rotators begin to participate in the job rotation and realize the knowledge exchange between the different positions through knowledge communicating. Each job is a knowledge base, and the employee on the position is a subset of the knowledge base. Employee participates in job rotation, and absorbs new tacit knowledge from the target rotating jobs, and then integrates the new knowledge with original personal knowledge system, so that they update the personal knowledge base. After the rotators return to the previous position, they could apply and create the tacit knowledge through complete the tasks and 
jobs on the core positions, and finally reflect in the output and results, to enrich and update the knowledge base and realize the transformation from personal knowledge to job knowledge (see Figure 1).

On the target position, the process that the rotator transfer the tacit knowledge with other employees contains knowledge sources output knowledge and knowledge receptors receive knowledge two links. The expressiveness of tacit knowledge and knowledge distance between knowledge transfer subjects will affect the process and efficiency of knowledge transfer. The knowledge distance means the differences degree of the knowledge structure between the knowledge subjects, which will be affected by the similarities of their respective jobs. The jobs of enterprises can be divided into different sequences, and the difference of tacit knowledge between job sequences is very big, which will lead to greater knowledge distance between employees and affect understanding and absorption of new knowledge. From tacit knowledge type and knowledge distance between knowledge transfer subjects two dimensions, the tacit knowledge transfer situation in the process of job rotation can be divided into four types (see Figure 2).

1) Situation one: pseudo tacit knowledge transfer between same job sequences.

The tacit knowledge transfer efficiency in the situation one is the highest. In the knowledge outputting link, knowledge source can express the pseudo tacit knowledge (e.g. work skills, the way of thinking, etc.) by description, explanation and demonstration to realize the tacit knowledge transfer indirectly through explicit knowledge transfer. In the knowledge receiving link, rotators rotate in the same job sequence, so that they will own the similar knowledge structure and cognitive background, which make it easier to establish shared mental model with employees in the target positions. The shared mental model refers to the knowledge structure shared by team members, which make the team members form the correct explanation of tasks. This can greatly promote the knowledge receiver's understanding, absorption and transformation of new knowledge,

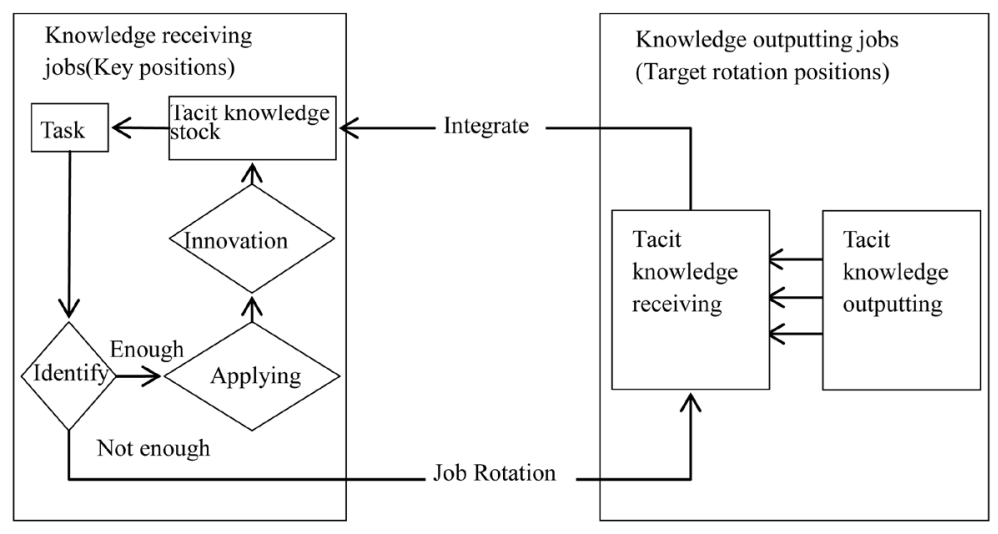

Figure 1. Tacit knowledge flow path during job rotation.

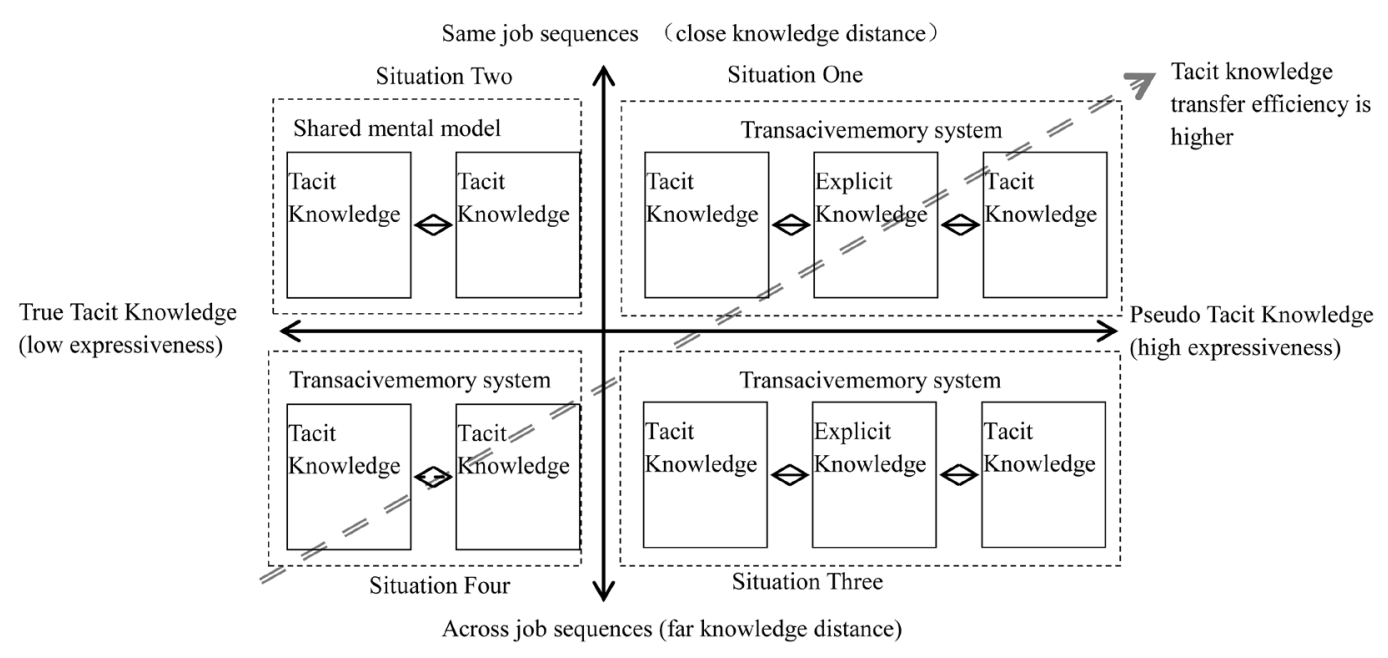

Figure 2. Two-dimensional situation coordinates. 
and help to achieve effective knowledge transfer. So it is easy for new knowledge recipient digest and memory, and finally fix the explicit knowledge to tacit knowledge through the practice in the process of the practical work and complete the tacit knowledge transfer process.

2) Situation two: true tacit knowledge transfer between same job sequences.

The tacit knowledge transfer efficiency in the situation two is the lower than the first situation, and the main difficulty states in the knowledge outputting link. Because true tacit knowledge (such as employees' mental models, group understanding, etc.) is hard to be expressed, but we can use metaphor and analogy to visualize the tacit knowledge, and the success of tacit knowledge transfer relies on the comprehension of the receiver. Due to the rotator rotate in the same job sequence, so that they will own a common working language and shared mental model with the target position employees. It is easy for the rotators to observe and imitate the knowledge carrier and form the cognitive for the tacit knowledge and further understand the intention and meaning of the actions to from personal understanding, and finally realize the directly transfer of tacit knowledge.

3) Situation three: pseudo tacit knowledge transfer across job sequences.

Tacit knowledge transfer efficiency in situation three is lower than the first and second situations, and the main difficulties lies in the knowledge receiving link. Due to the rotators rotate across the job sequences and different employees have their respective expertise knowledge, so that the two sides formed a transactive memory system emphasizing the knowledge division among employees, which will lead to more difficulties in knowledge understanding and absorption. In this situation, it is important to train knowledge receivers before implementing job rotation to establish the common knowledge base. In the process of knowledge transfer, knowledge source will code the pseudo tacit knowledge and assist knowledge receivers to edit and sort work skills, knowhow and thoughts to formwork behavior manual, case reports, etc., which is convenient to be stocked and memorized. Finally knowledge receivers need to take use of a lot of time and energy to practice repeatedly, trial, correct, and reflect in the practical work to on this process, to internalize the explicit knowledge gradually as personal skills, thinking mode, and individual tacit knowledge.

4) Situation four: true tacit knowledge transfer across job sequences.

Tacit knowledge transfer efficiency in the situation four is the minimum one, and both the tacit knowledge receiving link and outputting link face challenges. In this situation, the knowledge carriers are required good communication skills, at the same time, the recipient are required strong learning motivation and understanding ability. Besides that, close social relationships also need to be established between both sides. After a long time, highly frequent interaction, they could achieve enlightenment learning. During close interaction between the two sides, the knowledge receiver will observe, cognitive and imitate the knowledge carrier's words and actions, accompanied by a lot of face to face communication, to understand action connotation and target, and then after self-reflecting and grasping, the knowledge receiver will form a new cognition and comprehension, and then return to practical work to test, cycling the "cognitive-action-cognitive" process, until the knowledge recipient transfer the new knowledge into individual tacit knowledge, for their use.

\subsection{Tacit Knowledge Transfer Performance Evaluation Stage}

A sign of tacit knowledge transfer successfully is whether the knowledge transfer make up for the job knowledge gaps, and support the business process and help to realize organization's strategic goals. Enterprises can appraise the effect of knowledge transfer from three aspects: 1) the degree of support strategic goals. Whether the difference of the business performance indicators in the knowledge receiving department is in line with the enterprise strategic development direction should be considered firstly; 2) business process performance improvement. Rotate employees will integrate the new tacit knowledge into the working process, embodied in the business process performance improvement. So at the end of the job rotation, paying continuous attention to rotate employees' job performance could indirectly assess the effect of the recessive knowledge application and innovation; 3) the offset degree of the knowledge gap. At the end of the job rotation, reassess the tacit knowledge stock of the jobholder to see if the knowledge gap is made up.

\section{The Management Mechanism Using Job Rotation to Achieve Tacit Knowledge Transfer}

Implementing job rotation can realize individual tacit knowledge updating, and then rich and create the tacit knowledge in the key positions. But without a systematic management mechanism using job rotation to achieve 
tacit knowledge transfer, the implementation of job rotation will be lack of planning, sustainability, which is more likely to lead to deviate from the development goals, causing a waste of resources. So to design a set of effective management mechanism of tacit knowledge transfer could coordinate the tacit knowledge management goals and enterprise's business activities. Under the guidance of the enterprise strategic objectives, orderly implementing of job rotation could constantly enrich job knowledge, support business activities, gradually get close to the enterprise development goals, and then form a sustainable long-term operating mechanism. The management mechanisms using job rotation to achieve tacit knowledge transfer contains three specific sub-mechanisms: target sub-mechanism, dynamic sub-mechanism and feedback sub-mechanism (see Figure 3).

\subsection{Target Sub-Mechanism}

Enterprises need to develop long term and short term strategic development goals in order to adapt to the changes of external environment and realize their own sustainable development. Enterprises could start from the highest strategy target, decompose the overall goal and analysis the tacit knowledge gap to provide a clear direction for tacit knowledge management. If the employee's tacit knowledge reserve matches with the qualification of the key position, then it will support the operation of the core business process and also support the achievement of the enterprise strategic goals. If the incumbent's tacit knowledge doesn't match with the qualification of the core position and there exist knowledge gap, so they need to absorb new knowledge to be competent for this position. Target sub-mechanism unifies enterprise tacit knowledge transfer target and enterprise strategic development goals.

\subsection{Dynamic Sub-Mechanism}

In order to achieve the tacit knowledge transfer goals, enterprises need to provide a steady stream of power, prompting the fully interactive between the knowledge sources and receivers. Knowledge transfer power comes from the emergence of "knowledge gap". When the employees in key position are lack of knowledge and couldn't qualify the existing jobs, the demand of the knowledge transfer appears. Enterprise set knowledge transfer target, use appropriate incentives to promote knowledge transfer behavior between the knowledge transfer subjects, making the individual's needs get satisfied and at the same time realize the organizational goals. Companies

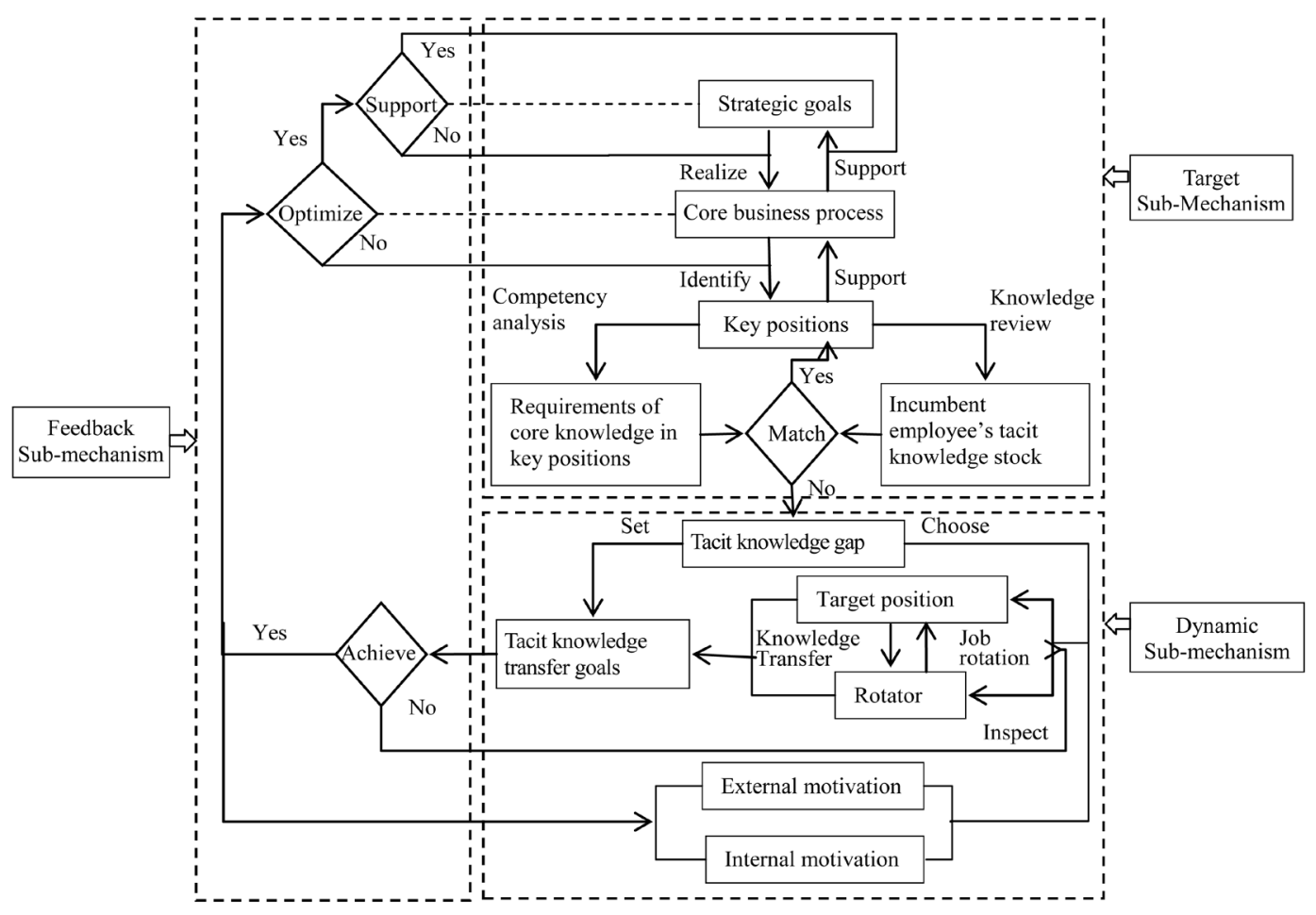

Figure 3. The management mechanisms using job rotation to achieve tacit knowledge transfer. 
can establish the performance evaluation system of knowledge contribution in the target positions and provide to knowledge contributor material rewards as external incentives, at the same time provide internal incentives to give employees a sense of achievement, to enhance the sense of identity and the respect from others. Besides that, enterprises could establish a performance evaluation system about knowledge learning, and provide them with more job opportunities and promotion opportunities, to affirm their self-improvement and self-growth of the employees, and further to stimulate the knowledge transfer will of knowledge carrier and learning motivation of rotators, which will provide a steady stream of power for the enterprise to achieve the goals of tacit knowledge transfer.

\subsection{Feedback Mechanism}

In order to prevent the knowledge transfer behavior or results deviate from the expected goal, enterprises need to take the appropriate method to intervene the key links in the knowledge transfer management system, making the system run in the right direction. In tacit knowledge management mechanism system, three key nodes need to be evaluated. Firstly, enterprise need to evaluate whether the tacit knowledge transfer needs are satisfied, namely whether the tacit knowledge increment and innovation make up the tacit knowledge gap. If the target is not reached, then enterprise needs to check job rotation process, and adjust target position or rotators. If the knowledge gap is made up, the next step is to assess whether the key business process performance is optimized or not, that is, whether the rotator has leant the tacit knowledge and apply it into the practical work. If this didn't happen, the reason may be the key position has been positioned in error, and enterprise needs to redefine key positions and analysis of the tacit knowledge gaps. If to this goal is reached, the third assessment phase is to analysis whether the enterprise strategic direction is supported. If the performance changes of the core business process doesn't align with the strategic direction, the reason may be due to mistakes are made in the core business process definition, and need to decompose the strategic target again; If the strategic development goals are supported, the tacit knowledge transfer process get successful.

Anyhow, in the management mechanism system using job rotation to realize tacit knowledge transfer, the target sub-mechanism ensure that the tacit knowledge transfer always pointing in the direction of the enterprise's strategic target; dynamic sub-mechanism provides accelerator for the tacit knowledge transfer through implementing job rotation motivating the knowledge subjects; finally the feedback sub-mechanism correct the deviation from the expected goal through intervening behavior and process, to ensure the cycle of tacit knowledge management mechanism can run smoothly, gradually enrich enterprise knowledge capital, and support the realization of strategic goals.

\section{Related Guarantee Measures}

There may be some obstacles in the running process of tacit knowledge transfer management mechanism, such as the enterprise hierarchy culture, closed communication environment, weak consciousness of knowledge management, staff's knowledge monopoly mentality and so on. These obstacle factors will weaken the will of knowledge transfer subjects, inhibit knowledge exchange between employees, and hinder efficient operation of the tacit knowledge transfer mechanism. So in order to provide a good environment for the tacit knowledge transfer mechanism running, enterprises should take the corresponding security measures:

\subsection{Cultivate Supportive Culture Environment}

In order to avoid hierarchy culture, selfish departmentalism, departments' clique, appearing in the organization and hindering knowledge exchanging between staff, enterprise should establish the culture of mutual benefit, mutual trust, knowledge sharing, encourage employees to achieve a win-win situation through knowledge sharing. At the same time, the enterprise needs to provide to employees hardware and soft environment to realize knowledge communication, good environment including office environment, such as setting the automatic office system, provides the Shared network, knowledge map, etc.; soft environment including relaxed human relationships atmosphere, such as informal communication and fellowship activities, helping staff to establish the harmonious interpersonal relationship, etc.

\subsection{Set up Knowledge Management System within the Enterprise}

Enterprises shall establish a knowledge management system, and stored knowledge unit in the database of the 
enterprise in a structured form, to make the enterprise internal tacit knowledge transparent. So that enterprise could identify which tacit knowledge is valuable, and position the location of the tacit knowledge, and describe the relationship among employees, positions and the tacit knowledge clearly, This helps companies to realize its own tacit knowledge stock, grasp enterprise tacit knowledge demand from macroscopic perspective, positioning tacit knowledge subject rapidly, so that to systematically implement job rotation to meet the needs of the enterprise development.

\section{Conclusion}

In summary, job rotation is an effective tool to transfer tacit knowledge within an enterprise, and the transferring process contains three stages: identifying the tacit knowledge transfer subjects, transferring process and evaluating tacit knowledge transfer performance. During this part, the efficiency of transferring tacit knowledge in different job rotation situations has been analyzed by two dimensions: knowledge type and knowledge distance. Finally, a mechanism to manage tacit knowledge transferring by implementing job rotation is constructed, including specific targeting sub-mechanism, dynamic sub-mechanism and feedback sub-mechanism. At the same time, some corresponding security measures are suggested for enterprises, in order to provide a good environment where the tacit knowledge transfer mechanism can run very well.

\section{References}

[1] Ribeiro, R. (2013) Tacit Knowledge Management. Phenomenology and the Cognitive Sciences, 12, 337-366. http://dx.doi.org/10.1007/s11097-011-9251-x

[2] Wang, G.H. and Chen, S.J. (2008) Research on Tacit Knowledge of Its Category and Model in Corporation. Scientific Management Research, 3, 79-82.

[3] Zheng, Z.L., Zhu, F.Q., Meng, Q.W. and Huang, F.Q. (2013) Research on Tacit Knowledge in the Sight of the Action: Based on Research of Polanyi's Tacit Knowledge and "the Shape of Actions". Studies in Science of Science, 10, 14531458 .

[4] Zhang, W. and Wang, Z.B. (2012) Study on Creating Enterprise Competitive Advantage from Tacit Knowledge. Forecasting, 6, 66-70.

[5] Li, J.H. and Chang, X.R. (2013) The Influence Factors of Knowledge Transfer: A Meta-Analytic Review. Studies in Science of Science, 3, 394-406.

[6] Li, Q. and Cheng, G. (2013) Summary of Researches on Enterprise Tacit Knowledge at Home and Abroad, Information Research. Information, 3, 29-34.

[7] Wang, J. and Jin, Z.M. (2004) Tacit Knowledge: The Wellspring to sustainable Competitive Advantage. Nankai Business Review, 5, 91-95.

[8] Li, Z.X., Wang, Q. and Qi, Y.X. (2006) Identify the Tacit Knowledge of Employees and Fuzzy Comprehensive Evaluation. Science and Technology Management Research, 5, 179-182.

[9] Yang, X.H. and Liu, Y. (2011) A Model Study on Tacit Knowledge Transfer in Enterprise Based on Talents Flow. The 7th China Science and Technology Policy and Management Academic Conference Proceedings, Nanjing, 22 October 2011, 1-10. 